\title{
Am Rande des Unendlichen: Numerische Verfahren für unbegrenzte Gebiete
}

\author{
Marcus J. Grote \\ Marcus Grote, 1966 in Göttingen geboren und in Genf aufgewachsen, studierte an \\ der Université de Genève und an der Stanford University, wo er 1995 bei Joseph \\ B. Keller promovierte. Nach zwei Forschungsjahren am Courant Institute of Mathe- \\ matical Sciences in New York wurde er 1997 zum Assistenz-Professor an die ETH \\ Zürich gewählt. Seine Forschungsinteressen liegen im Zusammenspiel zwischen der \\ Angewandten Mathematik und der Numerik von partiellen Differentialgleichungen.
}

Malgré moi l'infini me tourmente Alfred de Musset

\section{Einleitung}

Ein unendliches Gebiet gibt es in der Natur nur einmal, nämlich das Weltall. Demnach käme man leicht zum voreiligen Schluss, unbegrenzte Gebiete seien etwas Seltenes, was abgesehen von rechnergestützten Simulationen kollidierender schwarzer Löcher kaum Beachtung verdiene. Das Gegenteil ist der Fall: unbegrenzte Gebiete treten sehr häufig und bei den verschiedensten Anwendungen auf, die von der Radar- und Sonarerkennung über die Fluiddynamik und Erdbebensimulation bis in die Wetterforschung und Quantenchemie reichen. Tatsächlich ist meistens nur ein lokales Naturphänomen von Interesse, dessen Umgebung vielleicht im Umfang nicht wirklich unbegrenzt, aber doch so gross ist, dass die eigentliche Grenze kaum Einfluss hat. Ausserdem erleichtert eine

Ein wesentliches Instrument zur Lösung physikalischer Probleme mit Hilfe mathematischer Methoden ist die Modellbildung. Das zu behandelnde Problem wird dabei klassisch durch ein Modell beschrieben, das sich über ein begrenztes Gebiet erstreckt. In dem vorliegenden Beitrag illustriert M.J. Grote eindrucksvoll, wie es durch Einfügen eines künstlichen Randes und geschickt gewählter Randbedingungen gelingt, unbegrenzte Phänomene, wie z.B. Streufeldprobleme, mit Hilfe begrenzter Modelle zu beschreiben, welche dann zu Randwertaufgaben führen, die mit bekannten Methoden behandelt werden können. Dabei gilt es natürlich die künstlichen Randbedingungen so zu wählen, dass die Lösung des entsprechenden Randwertproblems mit der Lösung des ursprünglichen Problems im Unendlichen übereinstimmt. $j k$ 


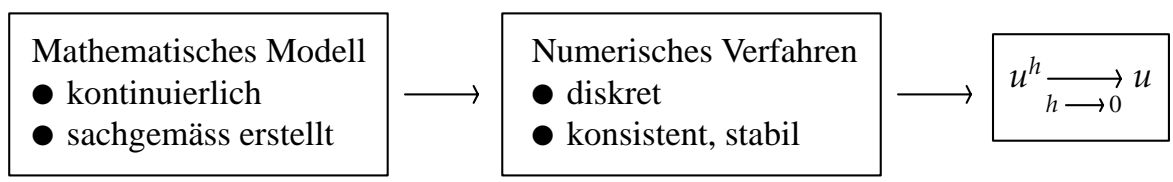

Abb. 1 Der klassische Vorgang bei der numerischen Approximation eines kontinuierlichen mathematischen Problems

abstrakte und vereinfachte Darstellung des Aussenraums unter Vernachlässigung zusätzlicher äusserer Nebeneffekte das isolierte Betrachten eines hochkomplexen, doch lokal begrenzten Phänomens. Man denke beispielsweise an die rechnergestützte Simulation der Luftströmung um einen Tragflügel beim Entwurf eines neuen Flugzeugs. Natürlich ist die Atmosphäre nur begrenzt, doch im Vergleich zur lokal bedingten Wechselwirkung des Luftstroms mit der Tragflügeloberfläche erscheint der Zustand der gesamten Atmosphäre nicht nur hochkompliziert, sondern auch ziemlich irrelevant. Ähnlich verhält es sich bei der numerischen Simulation einer Bohrinsel in Hinblick auf ihre Schwimmfähigkeit bei hohem Seegang, wobei es nicht nur überflüssig, sondern geradezu unmöglich ist, den gesamten Atlantik bis ins Detail auf dem Rechner darzustellen.

Mathematisch werden Naturphänomene durch partielle Differentialgleichungen dargestellt, deren Herleitung auf physikalischen Grundprinzipien (z.B. dem der Energieerhaltung) basiert. Viele Standardverfahren, wie zum Beispiel die Verfahren der finiten Differenzen und der finiten Elemente, ermöglichen die numerische Lösung von partiellen Differentialgleichungen. Ja, sie können sogar nichtlineares Verhalten erfassen und komplizierte Geometrien darstellen. Oft wird zunächst ein Gitter über das Rechengebiet $\Omega$ gelegt, an dessen Gitterpunkten die exakte Lösung $u$ durch die numerische Lösung $u^{h}$ angenähert wird. Erfüllt das numerische Verfahren gewisse Konsistenz- und Stabilitätsbedingungen, so erhält man generell ein konvergentes numerisches Verfahren, das mit immer kleiner werdender Maschenweite $h$ eine auch immer bessere Näherung der exakten Lösung liefert (Abb. 1).

Da all diese numerischen Verfahren jedoch davon ausgehen, das Rechengebiet sei begrenzt, fragt man sich natürlich, wie man sie denn auf ein unendliches Gebiet anwenden kann, und ferner, wie ein solches Gebiet auf einen "endlichen" Rechner passt. Dazu ist es offensichtlich notwendig, das Rechengebiet zuerst von seiner unbegrenzten Umgebung durch einen künstlichen Rand abzutrennen. Dies wirft jedoch sofort die rein mathematische Frage auf:

Welche Randbedingung am künstlichen Rand stellt sicher, dass die Lösung der Randwertaufgabe in dem endlichen Rechengebiet mit der Lösung des ursprünglichen Problems im Unendlichen übereinstimmt?

Finden wir solch eine Randbedingung, die den künstlichen Rand des Rechengebiets absolut transparent erscheinen lässt, so nennen wir sie exakt. Ansonsten entspricht sie nur einer angenäherten transparenten Randbedingung ${ }^{1)}$, deren künstliche Verzerrungen

1) “... also called radiating, absorbing, silent, transmitting, transparent, open, free-space, and one-way boundary conditions.”, Givoli, 1991 


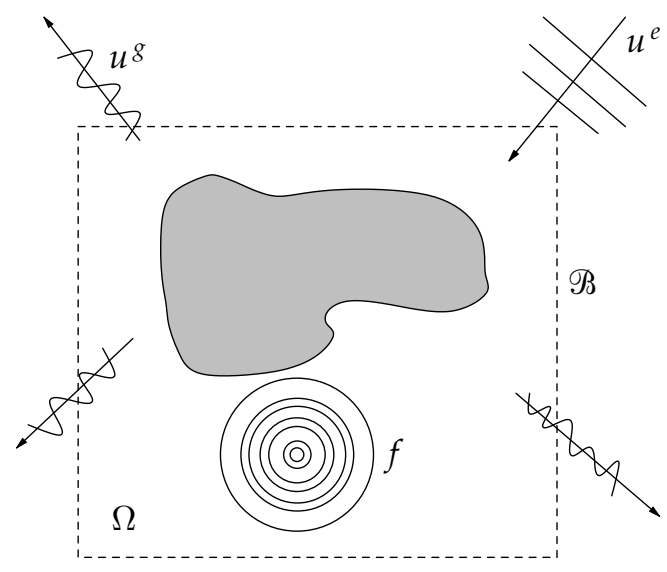

Abb. 2 Ein typisches Streufeldproblem besteht aus einem Hindernis, einem Quellterm $f$, einer einfallenden Welle $u^{e}$ und einer gestreuten Welle $u^{g}$. Der künstliche Rand $\mathscr{B}$ definiert den Rand des Rechengebiets $\Omega$.

die Lösung möglicherweise im gesamten Rechengebiet verfälschen. Dann besteht der Gesamtfehler der numerischen Lösung aus zwei unabhängigen Fehlerkomponenten: zum einen aus dem Approximationsfehler des numerischen Verfahrens und zum anderen aus dem Fehler am künstlichen Rand. Konvergenz innerhalb $\Omega$ zur ursprünglichen Lösung im unendlichen Gebiet kann nur erreicht werden, wenn beide Fehler systematisch verkleinert werden. Wird nur die Maschenweite $h$ verkleinert, ohne dem Fehler am Rand Beachtung zu schenken, so konvergiert das numerische Verfahren nicht gegen die ursprüngliche Lösung im Unendlichen, sondern gegen die Lösung einer anderen Randwertaufgabe innerhalb $\Omega$, welche die nur angenäherte Randbedingung erfüllt und daher jene am Rand erzeugten Verzerrungen enthält.

1.1 Streufeldprobleme. Da wir nicht auf all die zuvor erwähnten Anwendungen eingehen können, beschränken wir uns in diesem Aufsatz auf die Problemklasse der zeitabhängigen Streufeldprobleme. Im allgemeinen besteht ein Streufeldproblem aus einem Hindernis, einem Quellterm, und eventuell einer einfallenden Welle (Abb. 2). Ziel ist es zu berechnen, wie sich die gestreute Welle nach einer hochkomplexen, womöglich nichtlinearen, doch räumlich begrenzten Wechselwirkung ins Unendliche ausbreitet. Typische Anwendungen finden sich bei der Streuung von akustischen, elektromagnetischen und elastischen Wellen. Beispielsweise erfordert die numerische Simulation eines Mobilfunktelefons beim Entwurf einer optimalen Antenne die Lösung eines elektromagnetischen Streufeldproblems. Hierbei ist das Hindernis der menschliche Kopf, an dessen Ohrmuschel das Mobilfunktelefon, also der Quellterm $f$, gehalten wird. Die Verbindung zur Basisstation stellt eine ausgesandte elektromagnetische Welle her, die vom Kopf teils reflektiert, teils absorbiert wird. Durch eine optimale Form und Position der Antenne erreicht man eine minimale Deformation des Fernfeldes durch den Kopf, also eine maximale Rundstrahlcharakteristik, und einen niedrigen Energieverlust.

Als eines der ersten Verfahren, ein unbegrenztes Streufeldproblem innerhalb eines begrenzten Rechengebiets exakt zu berechnen, gilt der folgende Vorschlag von Smith 1974 
[14]. Sei $\Omega$ ein konvexes Gebiet in $\mathbb{R}^{2}$ oder $\mathbb{R}^{3}$, dessen äusserer Rand aus $n$ geraden Kanten (bez. ebenen Flächen in $\mathbb{R}^{3}$ ) besteht. Löst man die Anfangs- und Randwertaufgabe für jede Kombination von Dirichlet- und Neumann-Bedingungen ${ }^{2)}$ an den verschiedenen Kanten, so lässt sich die Lösung im Unendlichen als lineare Kombination jener $2^{n}$ Lösungen darstellen. Leider ist die Umsetzung von geringem praktischem Wert, da beispielsweise ein Würfel $2^{6}=64$ unabhängige numerische Lösungen erfordert, was vom Rechenaufwand und Speicherplatz her nur sehr schwer realisierbar ist. Es ist also nicht ausreichend zu zeigen, dass es ein mögliches Verfahren gibt, sondern das Verfahren muss sich auch in der Praxis vom Aufwand her bewähren. Aus diesem Grunde wurden bald danach die ersten approximativen Randbedingungen von Engquist und Majda [3] hergeleitet, die zwar nur angenähert gelten, sich jedoch ihrer Einfachheit wegen grosser Beliebtheit erfreuten (siehe Abschnitt 3.1). Diese ersten Randbedingungen sind heute noch weit verbreitet und basieren auf einer lokalen Zerlegung des Streufeldes in eindimensionale Wellen.

\section{Die eindimensionale Wellengleichung}

Als erstes befassen wir uns mit dem wohl einfachsten Streufeldproblem, der eindimensionalen Wellengleichung. Anhand dieses beinah trivialen Beispiels lassen sich viele Grundbegriffe erläutern; ausserdem lässt sich dann die exakte Randbedingung besonders einfach herleiten. Obwohl dieses Beispiel für didaktische Zwecke hervorragend geeignet ist, möchten wir vorweg darauf aufmerksam machen, dass die wirkliche Herausforderung erst in mehreren Dimensionen auftritt. In einer Dimension breitet sich nämlich eine Welle nur in zwei Richtungen aus, entweder nach links oder nach rechts. Sobald man jedoch mindestens zwei Dimensionen betrachtet, laufen von jedem Punkt unendlich viele Wellen aus, die sich auch in unendlich viele Richtungen ausbreiten.

Wir betrachten die eindimensionale Wellengleichung auf der unbegrenzten reellen Halbachse $x \geq 0$,

$$
\frac{\partial^{2} u}{\partial t^{2}}-\frac{\partial^{2} u}{\partial x^{2}}=f, \quad x>0, t>0
$$

und verlangen zusätzlich, dass beim Endpunkt $x=0$,

$$
u(0, t)=0, \quad t>0 .
$$

Man stelle sich zum Beispiel eine unendlich lange und dünne Gitarrensaite vor, die am linken Ende fest eingespannt ist und deren Auslenkung in Zeit und Ort vollständig durch die Funktion $u(x, t)$ beschrieben wird. Somit entspricht $u=0$ dem Ruhezustand. Falls sich die Saite nur leicht von ihrem Ruhezustand entfernt, beschreibt die eindimensionale Wellengleichung das Fortpflanzen einer Störung unter dem Einfluss der Kraft $f(x, t)$. Hierbei wurde in (1) durch Skalieren der Zeit die Ausbreitungsgeschwindigkeit auf eins normiert. Der Anfangszustand der schwingenden Saite wird durch Position und Geschwindigkeit bei $t=0$ vollständig bestimmt:

$$
u(x, 0)=U_{0}(x), \quad \frac{\partial}{\partial t} u(x, 0)=V_{0}(x), \quad x>0 .
$$

2) Bei der Dirichlet Randbedingung wird die Lösung und bei der Neumann Randbedingung ihre normale Ableitung auf Null gesetzt. 


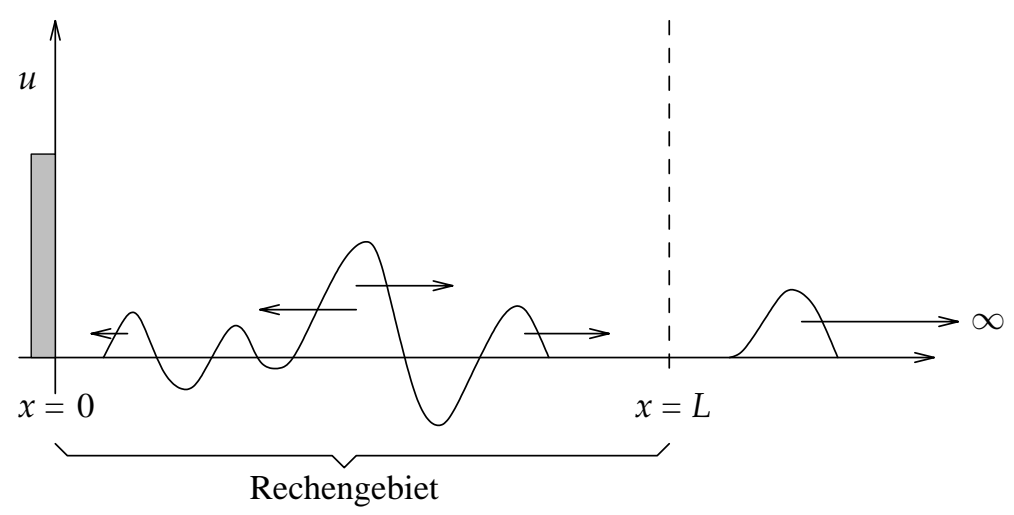

Abb. 3 Die eindimensionale Wellengleichung. Innerhalb des Rechengebietes $0 \leq x<L$ kann das Problem beliebig kompliziert sein, doch im Aussengebiet, $x \geq L$, werden $f=0$ für $t>0$, sowie $u=0$ und $\partial_{t} u=0$ bei $t=0$, angenommen.

Es lässt sich dann beweisen, dass die Anfangs- und Randwertaufgabe (1)-(3) auch sachgemäss gestellt ist: es existiert eine eindeutige Lösung, die von $U_{0}, V_{0}$, und $f$ stetig abhängt.

Den lokalen Charakter dieser Aufgabe bestimmt nun die folgende grundsätzliche Annahme: die Kraft wirke nur in einer begrenzten Umgebung der Verankerung bei $x=0$, so dass $f(x, t)=0$ ist für $x \geq L$ und $t>0$. Somit zerfällt die unendliche Halbachse in zwei Gebiete: zum einen das begrenzte Intervall $[0, L]$, und zum anderen das Intervall $[L, \infty)$, zwar unbegrenzt doch ohne Einwirken einer äusseren Kraft. Getrennt werden beide Bereiche durch die künstliche Grenze $\{x=L\}$, die hier nur aus einem einzigen Punkt besteht. Der Einfachheit halber nehmen wir zusätzlich an, dass zum Zeitpunkt $t=0$ die Saite sich im äusseren Bereich im absoluten Ruhezustand befindet: $U_{0}(x)=0$ und $V_{0}(x)=0$ für $x \geq L$. Unser Interesse gilt nun dem Intervall $[0, L]$, worin das Phänomen so kompliziert ist, dass der Einsatz des Rechners notwendig ist, um das zeitabhängige Verhalten der Lösung zu simulieren. Es wäre leicht, unser Modellproblem - zum Beispiel durch Hinzufügen nichtlinearer Effekte - weitaus komplizierter zu gestalten, und somit jeden Versuch einer rein analytischen Lösung zu vereiteln.

Wir können jedoch nicht einfach das numerische Verfahren unserer Wahl auf das Intervall $[0, L]$ beschränken und dabei den neuen künstlichen Randpunkt ignorieren. Ganz im Gegenteil, das Einführen des neuen Randpunktes bei $x=L$ erfordert unsere besondere Aufmerksamkeit. Rein mathematisch gesehen ist die Aufgabe (1)-(3), wenn auf $[0, L]$ beschränkt, doch ohne Randbedingung bei $x=L$, nicht sachgemäss gestellt, so dass dort eine Randbedingung notwendig ist. Um die Art und Weise dieser Randbedingung zu veranschaulichen und herzuleiten, muss man sich fragen, welche Rolle die gesuchte Randbedingung an der doch nur fiktiven Grenze $x=L$ spielt. Breitet sich eine Störung innerhalb $[0, L]$ nach rechts gegen $x=L$ aus, ist ihr sozusagen nicht bewusst, dass dieser Rand transparent ist und nur zum Schein das unendliche Aussengebiet abtrennt. Genau diese Information muss die Randbedingung der nach rechts auslaufenden Welle vermitteln, so dass jene ungehindert im künstlichen Rand verschwindet, als wäre er nicht vorhanden. Ansonsten würde ein Teil der Welle am künstlichen Rand reflektiert werden, 

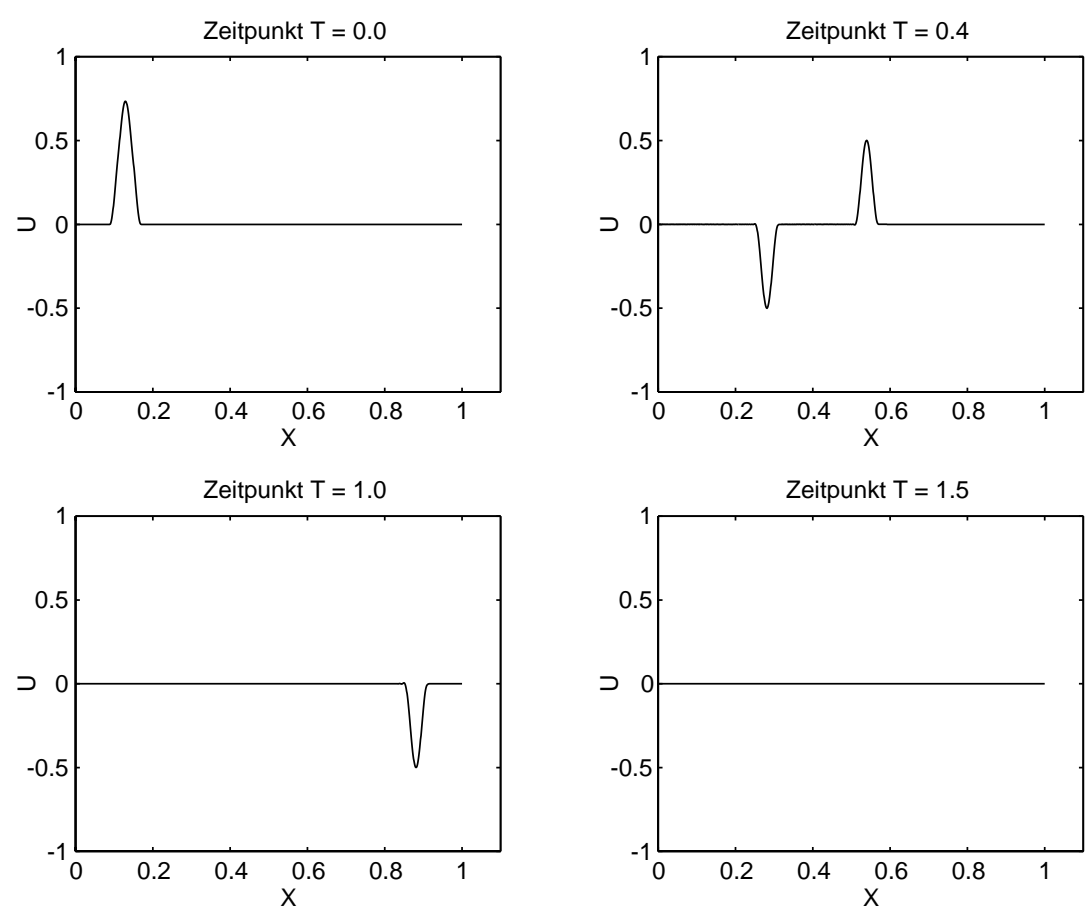

Abb. 4 Numerische Lösung der eindimensionalen Wellengleichung in einem halboffenen Gebiet: die Anfangsstörung zerfällt in zwei unabhängige Wellen, eine nach links und die andere nach rechts auslaufend. Die nach links laufende Welle trifft auf die 'Wand' bei $x=0$, wird gespiegelt und läuft dann der ersten nach, bis sie ebenfalls bei $x=1$ das Rechengebiet ungehindert verlässt.

zurück in das Rechengebiet laufen und die Lösung überall verzerren. Wie schon früher bemerkt läge diese etwaige Reflexion nicht an einem durch den Einsatz des Rechners unvermeidbaren Diskretisierungsfehler, sondern nur an der ungenauen Randbedingung. Falls wir eine Randbedingung finden, die keine unphysikalische Reflexion erzeugt und auslaufende Wellen ungehindert ins Unendliche weiterleitet, entspricht die Lösung innerhalb $\Omega$ mit jener Randbedingung bei $x=L$ genau der Lösung der ursprünglichen Aufgabe im unbegrenzten Gebiet. Demnach ist solch eine Randbedingung exakt.

Innerhalb des Rechengebiets $\Omega=[0, L]$ laufen die Wellen wie nach links so auch nach rechts. Im Aussengebiet hingegen existiert weder eine Kraft noch eine anfängliche Auslenkung. So kann dort keine neue Störung entstehen, und alle in $\Omega$ erzeugten Wellen laufen dort einfach ungehindert weiter nach rechts ins Unendliche, wie dargestellt in Abbildung 3. Um die exakte Randbedingung bei $x=L$ herzuleiten, müssen wir zuerst den einlaufenden Anteil der Welle vom dem auslaufenden Anteil trennen. Dazu definieren wir die Variablen $v$ und $w$,

$$
v=\frac{\partial u}{\partial t}+\frac{\partial u}{\partial x}, \quad w=\frac{\partial u}{\partial t}-\frac{\partial u}{\partial x}
$$


Da $u$ die Wellengleichung (1) erfüllt, sehen wir dass

$$
\frac{\partial v}{\partial t}-\frac{\partial v}{\partial x}=0, \quad \frac{\partial w}{\partial t}+\frac{\partial w}{\partial x}=0
$$

So lässt sich die Wellengleichung (1) als hyperbolisches System schreiben:

$$
\frac{\partial}{\partial t}\left[\begin{array}{c}
v \\
w
\end{array}\right]+\left[\begin{array}{cc}
-1 & 0 \\
0 & 1
\end{array}\right] \frac{\partial}{\partial x}\left[\begin{array}{c}
v \\
w
\end{array}\right]=0
$$

Die allgemeine Lösung von (4) ist

$$
v(x, t)=\phi(x+t), \quad \text { und } \quad w(x, t)=\psi(x-t),
$$

wobei $\phi$ und $\psi$ beliebige Funktionen einer Variablen sind, die durch Anfangs- und Randbedingungen bestimmt werden. Daraus folgt, dass $v$ konstant ist entlang Geraden der Form $x+t=c$, sogenannte charakterische Kurven, während $w$ entlang Geraden der Form $x-t=c$ unverändert bleibt. So erkennen wir in $v$ eine (nach links) einlaufende Welle, während $w$ einer (nach rechts) auslaufenden Welle entspricht. Um jede mögliche Reflexion bei $x=L$ zu vermeiden, darf keine einlaufende Welle existieren, das heisst wir müssen

$$
v(L, t)=0, \quad t>0,
$$

setzen. Anhand der Definition von $v$ folgt daraus die exakte (transparente) Randbedingung für die ursprüngliche Auslenkung der Gitarrensaite $u(x, t)$,

$$
\left(\frac{\partial}{\partial t}+\frac{\partial}{\partial x}\right) u=0, \quad x=L, \quad t>0
$$

In Abbildung 4 stellen wir unsere obige künstliche Randbedingung auf die Probe und verfolgen das zeitabhängige Verhalten einer Störung, die zuerst innerhalb $\Omega$ liegt. Sofort zerfällt die anfängliche Störung in zwei unabhängige Wellen, die sich in entgegengesetzte Richtungen ausbreiten. Während die eine Welle nach rechts läuft und bei $x=1$ das Rechengebiet verlässt, läuft die andere nach links, trifft auf die 'Wand' bei $x=0$, wird gespiegelt, und läuft dann der ersten nach, bis sie ebenfalls bei $x=1$ das Rechengebiet verlässt. Dank der exakten Randbedingung erscheint der rechte Rand des Rechengebiets bei $x=1$ vollkommen transparent, und beide Wellen verlassen das Rechengebiet ungehindert, unverzerrt und ohne jegliche unphysikalische Reflexion. Zum Schluss sei nochmal betont, dass die Problemstellung innerhalb $\Omega$ beliebig kompliziert sein darf. Dies hat keinen Einfluss auf die (exakte) transparente Randbedingung, deren Herleitung nur von den Eigenschaften im Aussenraum abhängt. 


\section{Wellenausbreitung in mehreren Dimensionen}

Dieser nächste Abschnitt führt uns in die weitaus kompliziertere Welt der mehrdimensionalen Wellenausbreitung. Ähnlich wie im vorherigen Abschnitt, betrachten wir ein lokales hochkomplexes Streufeldproblem, das in die unbegrenzte Ebene $\mathbb{R}^{2}$ eingebettet ist. Obwohl wir uns hier auf zwei Dimensionen im Raum beschränken, lässt sich die folgende Diskussion sofort auch auf drei Raumdimensionen übertragen. Um das lokale Phänomen von seiner unendlichen Umgebung abzutrennen, wählen wir eine in sich geschlossene Kurve, die unser Streufeldproblem umrandet. Ausserhalb dieser Kurve tritt weder ein Quellterm noch ein Hindernis auf, so dass jede Welle nach dem Überqueren dieser künstlichen Grenze sich gegen unendlich ausbreitet und nie wieder zurückkommt. Obwohl die Wahl dieser Kurve, also ihre Form und Position, uns frei steht, sollte sie konvex sein, um zu verhindern, dass eine Welle das Rechengebiet verlassen und später wieder eindringen kann. Ein in der Praxis seiner Einfachheit wegen bevorzugter Rand ist das Rechteck, da die Wellengleichung in cartesischen Koordinaten eine besonders einfache Schreibweise annimmt.

Wir betrachten nun die zweidimensionale Wellengleichung auf der unbegrenzten Ebene,

$$
\frac{\partial^{2} u}{\partial t^{2}}-\frac{\partial^{2} u}{\partial x^{2}}-\frac{\partial^{2} u}{\partial y^{2}}=f, \quad t>0
$$

mit entsprechenden Anfangsbedingungen

$$
u(x, y, 0)=U_{0}(x, y), \quad \frac{\partial}{\partial t} u(x, y, 0)=U_{1}(x, y), \quad t=0 .
$$

Wie zuvor sei das Phänomen hochkompliziert, womöglich nichtlinear, aber lokal. So lässt sich das Rechengebiet $\Omega$ von dem unendlichen Aussenraum durch einen künstlichen Rand abtrennen, den wir hier einfachheitshalber als das Quadrat $\mathscr{B}$ mit Eckpunkten $( \pm L, \pm L)$ wählen - siehe Abb. 2. Ausserhalb $\Omega$ nehmen wir an, dass weder eine anfängliche Störung noch eine äussere Kraft vorhanden sind:

$$
U_{0}(x, y)=U_{1}(x, y)=0, \quad \text { und } \quad f(x, y, t)=0, t>0, \quad(x, y) \in \mathbb{R}^{2} \backslash \Omega .
$$

Wieder stellt sich die Frage, welche Randbedingung für $(x, y) \in \mathscr{B}$ sicherstellt, dass die Wellen auch tatsächlich unbehelligt in den unendlichen Aussenraum gelangen, ohne jegliche ungewollte Reflexion zu erzeugen. Aus Symmetriegründen ist es ausreichend, wenn wir nur eine der vier Kanten des Rechtecks betrachten, hier die rechte Kante bei $x=L$. Somit liegen der Aussenraum rechts und das Rechengebiet $\Omega$ links des künstlichen Randes $\left\{(x, y) \in \mathbb{R}^{2} \mid x=L\right\}$. Da weder eine anfängliche Störung noch das Einwirken einer Kraft eine Welle im Aussenraum erzeugen können, breitet sich eine Welle im Gebiet $x \geq L$ immer gegen Osten aus, ohne je zurückzukommen. Damit die exakte transparente Randbedingung verhindert, dass etwaige Reflexionen bei $x=L$ entstehen, muss sie jede einkommende Welle auf Null setzen, natürlich ohne die auslaufenden Wellen in irgendeiner Weise zu beeinflussen. Dies konnten wir im letzten Abschnitt leicht erreichen und so die exakte Randbedingung für die eindimensionale Wellengleichung erzielen. Ganz anders ist es jedoch bei dem jetzigen zweidimensionalen Problem. Betrachten wir 
nämlich einen festen Punkt $(L, y)$ am künstlichen Rand, so treffen dort Wellen nicht nur aus einer, sondern aus unendlich vielen Richtungen ein, wenn auch nur aus Westen, Wellen, die sich dann auch in unendlich viele Richtungen fortpflanzen. Deshalb ist es hier auch wesentlich schwieriger, zwischen den einfallenden und den auslaufenden Wellen $\mathrm{zu}$ unterscheiden als in dem vorherigen eindimensionalen Beispiel.

Sei $\hat{u}(x, \xi, \omega)$ nun die Fouriertransformierte der Lösung $u(x, y, t)$ in der Tangentialebene parallel zum künstlichen Rand und in der Zeit,

$$
\hat{u}(x, \xi, \omega)=\int_{-\infty}^{\infty} \int_{-\infty}^{\infty} u(x, y, t) e^{i(\omega t+\xi y)} d y d t
$$

Hierbei wird in dem obigen Integral die Lösung $u(x, y, t)$ für $t<0$ auf Null gesetzt, damit sie für alle Zeit wohldefiniert ist. Dann lässt sich $u$ durch die entsprechende inverse Fouriertransformation von $\hat{u}$ darstellen, die der obigen Formel (nach Vertauschung von $u$ und $\hat{u}$ ) stark ähnelt. Da für $x \geq L$ die Lösung $u$ die Wellengleichung (6) mit $f=0$ erfüllt, erkennt man leicht, dass für ihre Fouriertransformierte gilt:

$$
\frac{\partial^{2}}{\partial x^{2}} \hat{u}=\left(\xi^{2}-\omega^{2}\right) \hat{u}, \quad x \geq L
$$

Um die exakte Randbedingung bei $x=L$ herzuleiten, möchten wir wie im eindimensionalen Fall eine Beziehung zwischen der ersten normalen Ableitung (hier nach $x$ ) von $u$ und womöglich anderen Ableitungen bezüglich der Tangentialvariablen (hier nach $y$ ) und der Zeit herleiten. Aus der Gleichung (7) geht hervor, dass $\partial_{x} \hat{u}$ durch $\pm \sqrt{\xi^{2}-\omega^{2}} \hat{u}$ bestimmt ist. Die Wahl des Vorzeichens der Wurzel entspricht genau der Unterscheidung zwischen ein- und auslaufenden Wellen, und man kann zeigen, dass die korrekte Wahl auf die folgende exakte Randbedingung führt:

$$
\frac{\partial}{\partial x} \hat{u}=-i \omega \sqrt{1-(\xi / \omega)^{2}} \hat{u}, \quad x=L .
$$

Obwohl der künstliche Rand bei $x=L$ dank dieser Randbedingung völlig transparent erscheint, besitzt diese Formulierung kaum einen praktischen Wert. In der Tat suchen wir ja keine Randbedingung für $\hat{u}$, sondern für $u$. Formal lässt sich die Fouriertransformation natürlich umkehren und dadurch $\partial_{x} u$ bestimmen. Anders als bei einem polynomialen Ausdruck, wo die inverse Fouriertransformierte auf einen lokalen Differentialoperator zurückführt, ergibt die Rücktransformation der obigen Randbedingung wegen der Wurzel jedoch keinen einfachen Differentialoperator. Stattdessen resultiert ein sogenannter Pseudo-Differentialoperator, der sich nur durch Vor- und Rücktransformation auswerten lässt. Die normale Ableitung $\partial_{x} u$ an einem festen Punkt $(L, y)$ hängt also global von der Gesamtauswertung von $u$ auf der Geraden $x=L$ und auf der gesamten Zeitachse $\mathrm{ab}$ und lässt sich weder in der Zeit noch im Ort lokal bestimmen.

“... unfortunately, these [perfectly absorbing] boundary conditions have to be nonlocal in both space and time”, Engquist \& Majda, 1977 
3.1 Angenäherte Randbedingungen. Eine Möglichkeit, die Schwierigkeiten der nichtlokalen Darstellung der exakten Randbedingung (8) zu überwinden, besteht darin, den obigen Pseudo-Differentialoperator durch einen lokalen angenäherten Differentialoperator zu approximieren. Dadurch gibt man die absolute Transparenz des Randes $\mathscr{B}$ auf und erklärt sich sozusagen bereit, einen bestimmten Anteil an künstlich erzeugter und unphysikalischer Reflexion in Kauf zu nehmen. Diesen Weg wählten Engquist und Majda 1977 [3], und wir gehen nun kurz auf diese Vorgehensweise ein, die sogar noch heute ihrer Einfachheit und Allgemeinheit wegen das wohl meistverbreitete Verfahren ist.

Aus der Fouriertransformation eines Differentialoperators resultiert im Frequenzbereich ein polynomialer Ausdruck, oft Symbol genannt. Beispielsweise erzeugt die Fouriertransformation des Differentialoperators $\partial_{y y}$ das Polynom $-\xi^{2}$. Diese Aussage gilt natürlich auch in entgegengesetzter Richtung, so dass jedes Polynom genau einem Differentialoperator entspricht. Finden wir also ein Polynom in $s=\xi / \omega$, das den Ausdruck $\sqrt{1-s^{2}}$ annähert, so wird dessen Rücktransformation einen Differentialoperator ergeben, der als angenäherte transparente Randbedingung eingesetzt werden kann.

Für genügend kleines $s$ können wir die Funktion $\sqrt{1-s^{2}}$ durch die ersten Terme der Taylor-Entwicklung annähern,

$$
\sqrt{1-s^{2}}=1-\frac{s^{2}}{2}+O\left(s^{4}\right), \quad|s| \rightarrow 0 .
$$

Ersetzen wir nun die Wurzel in (8) durch den Hauptterm der Taylor-Entwicklung, also $\sqrt{1-s^{2}} \simeq 1$, bekommen wir nach der Fourier-Rücktransformation

$$
\begin{aligned}
\partial_{x} \hat{u} \simeq & -i \omega \hat{u} \\
& \Rightarrow\left(\partial_{t}+\partial_{x}\right) u=0, \quad x=L .
\end{aligned}
$$

Dies ist die sogennante Engquist-Majda Randbedingung erster Ordnung, weil nur erste Ableitungen der Lösung vorkommen. Sie stimmt mit der exakten Randbedingung (5) für die eindimensionale Wellengleichung überein. Somit ist sie exakt für Lösungen der zweidimensionalen Wellengleichung, die nur von $x$ und $t$ abhängen, also Wellen, die normal am künstlichen Rand eintreffen. Verwenden wir die genauere Approximation $\sqrt{1-s^{2}} \simeq 1-s^{2} / 2$ in (8), so erreichen wir auf ähnliche Weise die Engquist-Majda Randbedingung zweiter Ordnung,

$$
\begin{aligned}
\partial_{x} \hat{u} \simeq & -i \omega\left(1-(\xi / \omega)^{2} / 2\right) \hat{u} \\
& \Rightarrow\left(\partial_{t t}+\partial_{t x}-\frac{1}{2} \partial_{y y}\right) u=0, \quad x=L .
\end{aligned}
$$

Diese Randbedingung ist ebenfalls exakt für normal einfallende Wellen, da wir sie durch Einsetzen der Wellengleichung (6) auch äquivalent als

$$
\left(\partial_{t}+\partial_{x}\right)\left(\partial_{t}+\partial_{x}\right) u=0, \quad x=L,
$$

umschreiben können. Weitere Approximationen der Funktion $\sqrt{1-s^{2}}$, sowohl durch rationale Funktionen (Padé-Approximationen) als auch mit Hilfe von TschebycheffPolynomen, die zusätzliche Terme der Taylor-Entwicklung miteinbeziehen und so auch 


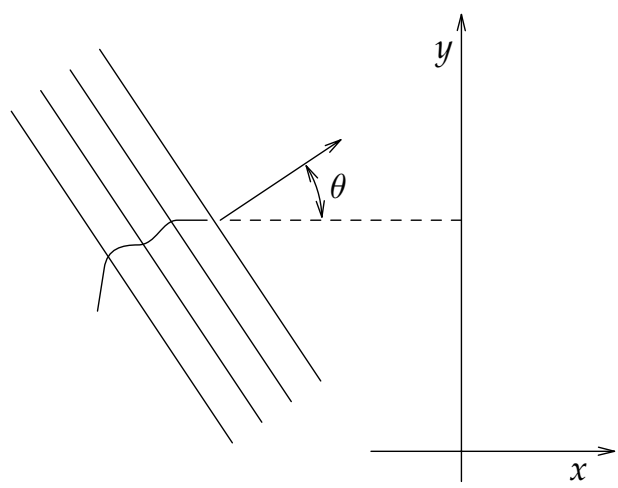

Abb. 5 Eine ebenene Welle trifft mit dem Einfallswinkel $\theta$ ein.

Ableitungen immer höherer Ordnung zur Folge haben, wurden dann auch vorgeschlagen und deren Absorptionseigenschaft in Hinblick auf transparente Randbedingungen wurde untersucht.

Schliesslich zeigte Higdon [10] jedoch, dass jede dieser verschiedenen Randbedingungen als Spezialfall der folgenden Klasse von Randoperatoren zu verstehen ist, wobei $\alpha_{1}, \ldots, \alpha_{p}$ feste Parameterwerte sind:

$$
\left(\cos \alpha_{p} \frac{\partial}{\partial t}+\frac{\partial}{\partial x}\right) \ldots\left(\cos \alpha_{1} \frac{\partial}{\partial t}+\frac{\partial}{\partial x}\right) u=0, \quad x=L
$$

Zum Beispiel entspricht die zweite Engquist-Majda Randbedingung (9) der Higdonschen Formel (10) mit $\alpha_{1}=0^{\circ}$ und $\alpha_{2}=0^{\circ}$. Diese verallgemeinerte Formulierung veranschaulicht auf vortreffliche Weise, inwiefern der Einsatz der Randbedingung vermag, den künstlichen Rand transparent erscheinen zu lassen. Der gesamte Randoperator erscheint nämlich jetzt als Produkt von Differentialoperatoren erster Ordnung der Form $\cos \alpha_{i} \partial_{t}+\partial_{x}$. Da solch ein Differentialoperator einlaufende Wellen je mit Einfallswinkel $\pm \alpha_{i}$ am künstlichen Rand exakt auslöscht, folgt daraus, dass der gesamte Randoperator als Produkt an den diskreten Einfallswinkeln $\alpha_{1}, \ldots, \alpha_{p}$ keine künstliche Reflexion erzeugt. Die Wahl der Parameter $\alpha_{1}, \ldots, \alpha_{p}$ steht frei und kann dem Problem angepasst werden.

Nichtsdestoweniger sind all diese Randbedingungen nur Näherungen der exakten Randbedingung (8), und demnach erzeugen sie bei $x=L$ einen gewissen Anteil an unphysikalischer Reflexion. Wie gross ist dieser Anteil an künstlicher Reflexion für eine bestimmte Randbedingung? Um dies zu beantworten, erinnern wir uns an das Überlagerungsprinzip: jede Lösung der (homogenen) Wellengleichung lässt sich als Überlagerung von ebenen Wellen darstellen. Wir betrachten nun in Abbildung 5 eine Familie von (eindimensionalen) ebenen Wellen, die mit einem Einfallswinkel $\theta$ am künstlichen Rand $x=L$ von links her eintreffen. Aus der Linearität der Wellengleichung (6) und der Randbedingung (10) lässt sich leicht zeigen, dass eine bei $x=L$ reflektierte Welle mit derselben Frequenz wieder in das Rechengebiet zurückläuft. Daher besteht die Lösung aus einer auslaufenden Welle, deren Amplitude wir auf eins normieren, und einer künstlich erzeugten 


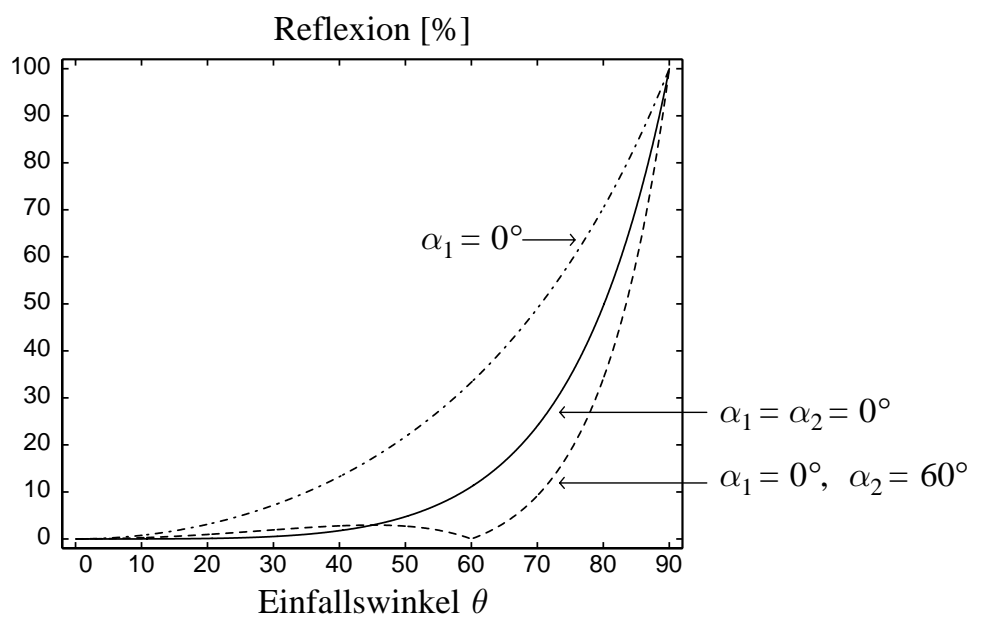

Abb. 6 Der prozentuale Anteil an künstlicher Reflexion bei einer ebenen Welle mit Einfallswinkel $\theta$ (11), den der Einsatz der angenäherten Randbedingung (10) erzeugt.

einlaufenden Welle mit Amplitude $|r|$ :

$$
u(x, y, t)=e^{i(k x+\ell y-\omega t)}+r e^{i(-k x+\ell y-\omega t)}, \quad k, \omega \geq 0,
$$

Hier ist $r=r\left(\theta ; \alpha_{1}, \ldots, \alpha_{p}\right)$ eine Funktion des Einfallswinkels $\theta$ (durch $\tan \theta=\ell / k$ bestimmt) und der festen Parameter $\alpha_{1}, \ldots, \alpha_{p}$. In Abbildung 6 untersuchen wir drei angenäherte Randbedingungen auf ihre Absorptionseigenschaft, indem wir den reflektierten Anteil $|r|$ bezüglich des Einfallswinkels $\theta$ aufzeichnen. Die Randbedingung mit $\alpha_{1}=0^{\circ}$ entspricht der ersten, und die Randbedingung mit $\alpha_{1}=0^{\circ}$ und $\alpha_{2}=0^{\circ}$ entspricht der zweiten Engquist-Majda Randbedingung. Eine weitere beliebte Wahl entspricht den Werten $\alpha_{1}=0^{\circ}$ und $\alpha_{2}=60^{\circ}$, welche einlaufende Wellen, die normal oder mit einem Einfallswinkel von $60^{\circ}$ den künstlichen Rand erreichen, exakt auslöscht. Für andere beliebige Einfallswinkel, also $\theta \neq 0^{\circ}$ und $\theta \neq 60^{\circ}$, ergibt sich ein gewisser Anteil an unphysikalischer Reflexion, der bei fast normalem Einfallswinkel sehr klein ist, doch mit wachsender Abweichung der Einfallsrichtung von der Normalen zum Rand rasch zunimmt.

3.2 Exakte Randbedingungen. Eine Alternative zur lokalen Approximation der exakten Randbedingung durch einen angenäherten Differentialoperator bietet die Kirchhoffsche Integraldarstellung der exakten Lösung [13]. Nach dem Huygens'schen Prinzip [11] löst jeder Punkt am künstlichen Rand $\mathscr{B}$ zu jedem Zeitpunkt eine Kugelwelle aus,

“... jede kleine Stelle eines leuchtenden Körpers, wie der Sonne, einer Kerze oder einer glühenden Kohle, ihre Wellen erzeugt, deren Mittelpunkt diese Stelle ist.”, $\quad$ Huygens, 1690

und die Überlagerung aller Kugelwellen ergibt das Streufeld im Aussenraum. Die Kirchhoffsche Integralformel ist eine analytische Umsetzung des rein geometrischen Huygens'schen Prinzips. Dadurch kann die Lösung zu jeder Zeit und in jedem Punkt im 
Aussenraum, also auch am künstlichen Rand $\mathscr{B}$, als Faltungsintegral über die gesamte Vergangenheit der Lösung auf $\mathscr{B}$ dargestellt werden. Leider ist die numerische Auswertung der Faltungsintegrale so aufwendig, dass dieses Verfahren in der Praxis kaum Anwendung findet. In drei Raumdimensionen zum Beispiel muss für jeden Punkt $x \in \mathscr{B}$ und zu jedem Zeitpunkt $t>0$ ein Faltungsintegral in Zeit und Ort auf der Schnittmenge des aus $(x, t)$ ausgehenden Lichtkegels und des künstlichen Randes, also auf einer zweidimensionalen Mannigfaltigkeit, ausgewertet werden. Ist $N$ ein Mass für die Anzahl der Gitterpunkte in einer Raumdimension, so erfordert ein numerisches Standardverfahren pro Zeitschritt einen Rechenaufwand, der wie $N^{3}-$ die Anzahl der Gitterpunkte in $\Omega$ - wächst. Dagegen verlangt die Auswertung der zweidimensionalen Faltungsintegrale an den $N^{2}$ Randpunkten pro Zeitschritt einen zusätzlichem Rechenaufwand, der wie $N^{2} \times N^{2}=N^{4}$ wächst. Ausserdem benötigt dieses Verfahren einen erheblichen Speicherplatz, da die Vergangenheit der Lösung am künstlichen Rand gespeichert werden muss.

So standen Anfang der 90er Jahre zwei Verfahren zur numerischen Lösung von zeitabhängigen Streufeldproblemen in unbegrenzten Gebieten zur Verfügung: zum einen die lokalen angenäherten Randbedingungen, deren Einsatz zwar einfach, doch immer mit Verzerrungen durch unphysikalische Reflexion am Rand verbunden ist, und zum anderen exakte Randbedingungen, deren numerische Umsetzung einfach zu aufwendig ist. Mit dem wachsenden Bestreben der Natur- und Ingenieurswissenschaften nach naturgetreuem Realismus bei der rechnergestützten Simulation, eine Entwicklung, die von den rasanten Fortschritten der Computerindustrie profitiert, wächst auch der Anspruch auf erhöhte Genauigkeit bei der realistischen, zeitabhängigen und dreidimensionalen numerischen Simulation. Bei der Entwicklung numerischer Verfahren könnte man daher zunehmende Genauigkeit und Anpassungsfähigkeit erwarten. Deswegen ist es besonders frustrierend, wenn all die neuesten Errungenschaften numerischer Verfahren, beispielsweise Adaptivität und erhöhte Konvergenzordnung, durch unphysikalische Reflexionen am Rand zunichte gemacht werden.

Ein Ausweg aus diesem Dilemma bot die Herleitung einer zeitlokalen und exakten Randbedingung für die Wellengleichung in drei Raumdimensionen für den Fall eines sphärischen künstlichen Randes [5], dessen Form und Position ja frei zur Wahl stehen. Die numerische Umsetzung dieser neuartigen Formulierung, die ohne Faltungsintegrale in der Zeit auskommt und so nur wenig zusätzlichen Aufwand benötigt, bestätigte auch deren vorteilhafte praktische Eigenschaften [6]. Solche exakten Randbedingungen lassen sich auch für die Streuung von elektromagnetischen oder elastischen Wellen herleiten [7, 8], worauf wir hier jedoch nicht eingehen. Stattdessen vergleichen wir im nächsten Abschnitt anhand eines konkreten numerischen Beispiels lokale angenäherte Randbedingungen mit den eben erwähnten (zeitlokalen) exakten Randbedingungen.

\section{Numerisches Beispiel}

In diesem Abschnitt vergleichen wir die Genauigkeit verschiedener Randbedingungen anhand eines numerischen Testbeispiels. Folgende Fragen sind von besonderem Interesse: Wie genau sind exakte Randbedingungen, wenn sie tatsächlich in der Praxis eingesetzt - und dabei natürlich auch numerisch angenähert - werden? Wie verhält sich die numerische Lösung, wenn die Maschenweite verkleinert wird? Konvergiert sie gegen die 


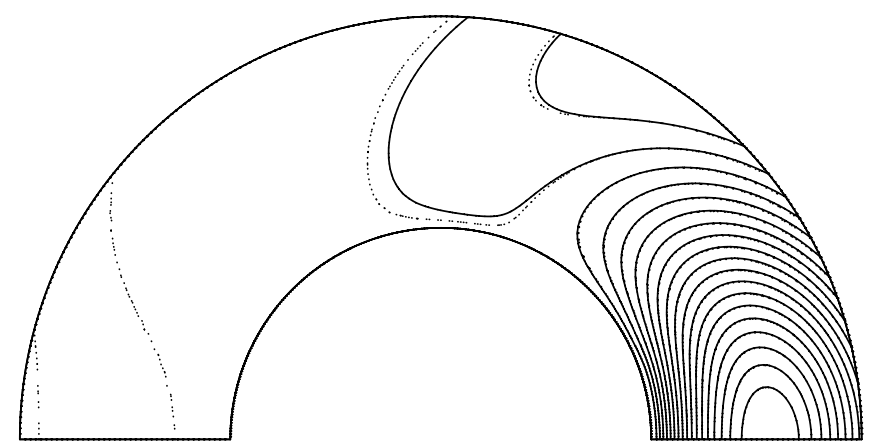

Abb. 7 Kolben auf einer Kugeloberfläche: die exakte Lösung $U_{\infty}^{h}(-)$ im unbegrenzten Aussenraum wird mit der angenäherten Lösung $U^{h}(\ldots)$ verglichen, die innerhalb $\Omega$ mit der lokalen Randbedingung BT2 am künstlichen Rand berechnet wurde.

exakte Lösung des ursprünglichen Problems im unbegrenzten Gebiet? Lohnt sich der zusätzliche Aufwand bei der Anwendung einer exakten Randbedingung, oder reichen lokale angenäherte Randbedingungen nicht doch aus?

Als Testbeispiel betrachten wir einen kreisförmigen Kolben auf einer Kugeloberfläche mit Radius $r_{0}=0.5$. Der Kolben befindet sich über dem Nordpol zwischen $0^{\circ} \leq$ $\theta \leq 15^{\circ}$. Durch radiale Vibrationen der Geschwindigkeit $\sin (\omega t)$ wird ein akustisches zeitabhängiges Streufeld erzeugt, das sich um die gesamte Kugel entfaltet und sich bis ins Unendliche ausbreitet. Ausserhalb des Kolbenbereichs verschwindet das akustische Streufeld an der Kugeloberfläche. Eine grosse Herausforderung bei diesem Testbeispiel besteht darin, dass die Wellen, die am Nordpol $\theta=0^{\circ}$ entstehen, entlang Längenkreisen auseinanderlaufen und dann stark abgeschwächt am Südpol wieder aufeinandertreffen. Somit ist die Amplitude der Wellen im Bereich hinter der Kugel weit geringer als in der Nähe des Kolbens.

Da dieses Problem axialsymmetrisch ist, bleibt das akustische Streufeld entlang Breitengraden konstant und ist daher unabhängig von $\phi$. So kann das Problem auf eine Schnittfläche $\phi=$ konstant reduziert und zweidimensional behandelt werden, was den Rechenaufwand erheblich verringert. Um den unendlichen Aussenraum abzutrennen, setzen wir bei $r=1$ einen künstlichen Rand $\mathscr{B}$ ein. Innerhalb des Rechengebietes $\Omega$, das zwischen der physischen Kugel bei $r_{0}$ und dem künstlichen Rand bei $r=1$ liegt, wird die numerische Lösung auf einem eher feinen Gitter mit 40 Punkten in der radialen und 240 Punkten in der Nord-Süd Richtung diskretisiert. Im Aussenraum setzen wir die Schallgeschwindigkeit auf eins und die Vibrationsfrequenz des Kolbens auf $\omega=2 \pi$. Obwohl wir die exakte Lösung im gesamten Aussenraum nicht kennen, können wir sie numerisch in $\Omega$ bis zu einem festen Zeitpunkt annähern, indem wir das Gitter nach aussen bis $r=R$ erweitern. Da sich die Wellen mit endlicher Geschwindigkeit ausbreiten, wird die numerische Lösung innerhalb $\Omega$ in völliger Unkenntnis des künstlichen Randes bis zu dem Zeitpunkt berechnet, wo die Wellenfront bei $r=R$ angelangt und wieder nach $\Omega$ zurückgekehrt ist. Diese Verfahrensweise ist natürlich mit einem sehr grossen Rechenaufwand verbunden und wäre in einer realen drei-dimensionalen Situation kaum denkbar. Wir bezeichnen diese numerische Lösung mit $U_{\infty}^{h}$, da sie innerhalb $\Omega$ und für 

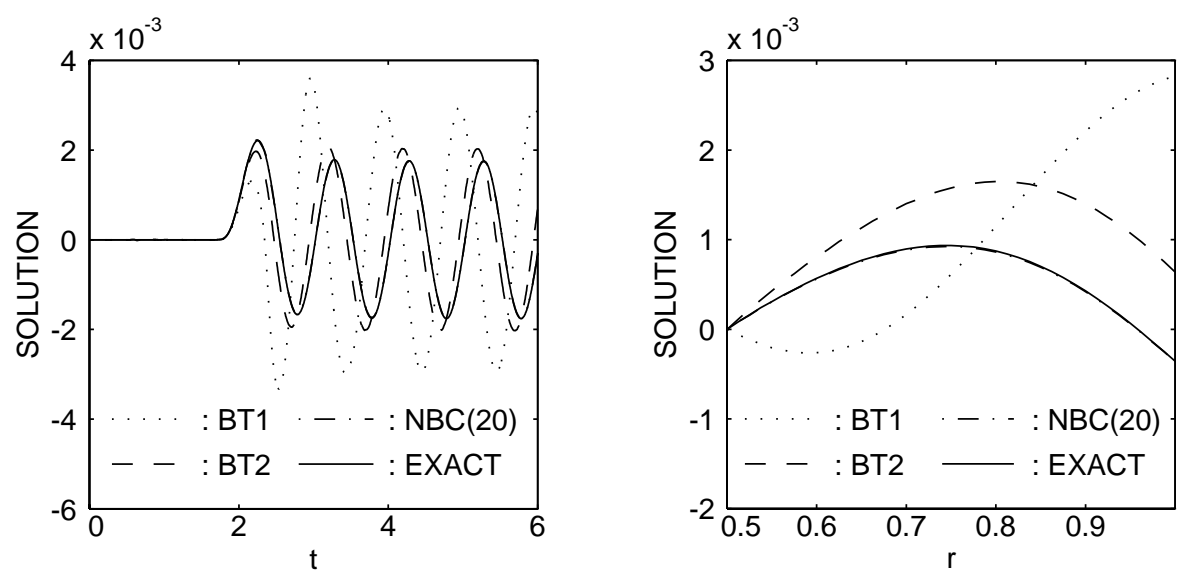

Abb. 8 Kolben auf einer Kugeloberfläche. Links: die numerischen Lösungen $U^{h}$, jeweils mit den Randbedingungen BT1, BT2, und NBC am künstlichen Rand berechnet, werden mit der exakten Lösung $U_{\infty}^{h}$ an einem festen Beobachtungspunkt bezüglich der Zeit verglichen. Rechts: dasselbe beim Zeitpunkt $t=10$ entlang der $z$-Achse unter dem Südpol, mit $\theta=180^{\circ}$ und $0.5 \leq r \leq 1$.

endliche Zeit der numerischen Lösung im unendlichen Gebiet entspricht. Zu unserem Zweck genügt die Wahl $R=6$, was das Ausrechnen von $U_{\infty}^{h}$ bis zum Zeitpunkt $t=10.5$ ermöglicht.

Am künstlichen Rand $\mathscr{B}$ bei $r=1$ möchten wir nun drei verschiedene Randbedingungen einsetzen und vergleichen. Zum einen die lokalen Randbedingungen BT1 und BT2 erster und zweiter Ordnung von Bayliss und Turkel [1], und zum anderen die exakte Randbedingung $\mathrm{NBC}^{3)}$, die in [5] hergeleitet wurde. Die Randbedingung BT1 gleicht der ersten Randbedingung von Engquist und Majda, und BT2 ähnelt stark der entsprechenden Randbedingung zweiter Ordnung - siehe Abschnitt 3.1. Beim Betrachten der Konturlinien der numerischen Lösungen beim Zeitpunkt $t=10$ (Abb. 7) fällt auf, dass die mit BT2 gerechnete Lösung im Bereich des Kolbens mit $U_{\infty}^{h}$ beinahe übereinstimmt. Richten wir unsere Aufmerksamkeit jedoch auf den Bereich hinter der Kugel, bemerken wir eine gegen den Südpol laufende unphysikalische Reflexion, die durch BT2 künstlich bei $\mathscr{B}$ erzeugt wurde. Dagegen stimmen die Konturlinien der numerischen Lösung, bei der NBC eingesetzt wurde, im gesamten Rechengebiet mit denen von $U_{\infty}^{h}$ so genau überein, dass sie hier voneinander nicht zu unterscheiden sind.

Da die künstliche Reflexion besonders in der Nähe des Südpols auffällt, konzentrieren wir uns weiter auf diesen Bereich. Als erstes vergleichen wir das Verhalten der Lösungen $U^{h}$ und $U_{\infty}^{h}$ bezüglich der Zeit beim Punkt $\theta=180^{\circ}$ und $r=1$, also unterhalb des Südpols auf dem künstlichen Rand (Abb. 8). Die drei Lösungen $U^{h}$ entsprechen den Randbedingungen BT1, BT2 und NBC. Neben dem zeitabhängigen Verhalten betrachten wir auch dieselben Lösungen beim Zeitpunkt $t=10$ entlang der $z$-Achse, $0.5 \leq r \leq$ 1 und $\theta=180^{\circ}$, die unterhalb der Kugel herausragt (Abb. 8). Beide Lösungen $U_{\infty}^{h}$ und $U^{h}$, die mit NBC gerechnet wurden, lassen sich kaum voneinander unterscheiden.

3) Nonreflecting Boundary Condition 
Dagegen weichen beide Lösungen $U^{h}$, die durch Einsatz der lokalen Randbedingungen BT1 und BT2 erzielt wurden, stark von der exakten Lösung $U_{\infty}^{h}$ ab. Die Reflexionen, die beide lokale Randbedingungen am künstlichen Rand erzeugen, laufen zurück in das Rechengebiet und verfälschen die numerische Lösung bis an das Hindernis heran. Hierbei möchten wir nochmals betonen, dass diese Reflexionen nicht an etwaigen numerischen Diskretisierungsfehlern liegen, sondern ganz allein auf die Ungenauigkeit der lokalen angenäherten Randbedingungen zurückzuführen sind.

\section{Abschliessende Bemerkungen}

Zum Abschluss dieses Artikels sei hervorgehoben, dass er nicht den Anspruch auf eine vollständige oder endgültige Darstellung der transparenten Randbedingungen erhebt. Insbesondere wurde der im Ingenieurswesen beliebte dritte Weg der absorbierenden Schichten $[2,12]$ bei der Behandlung der lokalen angenäherten und der exakten Randbedingungen völlig ausser acht gelassen. Auch die mathematisch komplizierte Frage, ob die Randwertaufgabe in dem Rechengebiet mit der transparenten Randbedingung sachgemäss gestellt sei, wurde hier stillschweigend übergangen. Die Beantwortung dieser Fragen verlangt nach aufwendigen analytischen Werkzeugen [9] und steht bei vielen neuartigen Randbedingungen noch offen.

Es versteht sich von selbst, dass die Vielfalt der Anwendungen es unmöglich macht, hier mehr als einen kleinen Einblick in das Gebiet der numerischen Verfahren im Unendlichen zu geben. Das wachsende Verlangen nach immer höherer Genauigkeit und die jüngsten Entwicklungen auf dem Feld der numerischen Verfahren stellen auch immer höhere Anforderungen an die transparenten Randbedingungen. Besonders reizvoll ist hierbei für den Autor das Zusammenspiel zwischen analytischen und numerischen Verfahren - erst ihr gemeinsamer Einsatz ermöglicht es, auf dem Feld der unbegrenzten Gebiete Fortschritte zu erzielen.

Danksagung: Dieser Artikel basiert auf meiner Einführungsvorlesung an der ETH Zürich vom April 1998. Ich möchte Herrn Professor Urs Stammbach für die Einladung danken, das Manuskript der Vorlesung für die Elemente der Mathematik zu überarbeiten.

\section{Literatur}

[1] A. Bayliss und E. Turkel, "Radiation boundary conditions for wave-like equations", Comm. Pure Appl. Math. 33 (1980), 707-725.

[2] J.-P. Bérenger, "A perfectly matched layer for the absorption of electromagnetic waves", J. Comput. Phys. 114 (1994), 185-200.

[3] B. Engquist und A. Majda, "Absorbing boundary conditions for the numerical simulation of waves", Math. Comp. 31 (1977), 629-651.

[4] D. Givoli, "Non-reflecting boundary conditions", J. Comput. Phys. 94 (1991), 1-29.

[5] M. J. Grote und J. B. Keller, "Exact nonreflecting boundary conditions for the time dependent wave equation”, SIAM J. Appl. Math. 55 (1995), 280-297.

[6] M. J. Grote und J. B. Keller, "Nonreflecting boundary conditions for time dependent scattering", J. Comput. Phys. 127 (1996), 52-65.

[7] M. J. Grote und J. B. Keller, "Nonreflecting boundary conditions for Maxwell's equations", J. Comput. Phys. 139 (1998), 327-342. 
[8] M. J. Grote und J. B. Keller, "Exact nonreflecting boundary condition for elastic waves", SIAM J. Appl. Math., im Druck.

[9] B. Gustafsson, H.-O. Kreiss, und J. Oliger, "Time Dependent Problems and Difference Methods", John Wiley \& Sons, New York, 1995.

[10] R. L. Higdon, "Absorbing boundary conditions for difference approximations to the multi-dimensional wave equation", Math. Comput. 47 (1986), 437-459.

[11] C. Huygens de Zuilichem, "Traité de la Lumière, où sont expliquées les causes de ce qui luy arrive dans la réflexion, dans la réfraction, et particulièrement dans l'étrange réfraction du cristal d'Islande", chez P. van der Aa, Leide, 1690. (“Abhandlung über das Licht", Ostwald's Klassiker der exakten Wiss. Nr. 20, Hg. von W. Engelmann, Leipzig, 1913.)

[12] M. Israeli und S. A. Orszag, "Approximation of radiation boundary conditions", J. Comput. Phys. 41, pp. 115-135, 1981.

[13] G. Kirchhoff, "Zur Theorie der Lichtstrahlen", Sitzber. d. k. Acad. d. Wissensch. zu Berlin vom 22. Juni 1883, p. 641. Wied. Ann. Bd. 18, p. 663, 1883.

[14] W. D. Smith, "Nonreflecting plane boundary for wave propagation problems", J. Comput. Phys. 15 (1974), 492-503.

Marcus J. Grote

Departement Mathematik

ETH Zentrum

CH-8092 Zürich 